\title{
BIRTH-DEATH PROCESSES WITH KILLING
}

\author{
Erik A. van Doorn* and Alexander I. Zeifman ${ }^{\dagger}$
}

*Department of Applied Mathematics

University of Twente

P.O. Box 217, 7500 AE Enschede, The Netherlands

E-mail: e.a.vandoorn@utwente.nl

\author{
${ }^{\dagger}$ Vologda State Pedagogical University \\ and Vologda Scientific Coordinate Centre of CEMI RAS \\ S. Orlova 6, Vologda, Russia \\ E-mail: zai@uni-vologda.ac.ru
}

9 December 2004

\begin{abstract}
The purpose of this note is to point out that Karlin and McGregor's integral representation for the transition probabilities of a birth-death process on a semi-infinite lattice with an absorbing bottom state remains valid if one allows the possibility of absorption into the bottom state from any other state. Conditions for uniqueness of the minimal transition function are also given.
\end{abstract}

Keywords and phrases: Karlin-McGregor representation, orthogonal polynomials, transition function, transition probabilities, state-dependent killing rate, total catastrophe

2000 Mathematics Subject Classification: Primary 60J80 


\section{Introduction}

We consider a Markov chain $\mathcal{X} \equiv\{X(t), t \geq 0\}$ taking values in $S \equiv\{0,1, \ldots\}$ with $q$-matrix $Q \equiv\left(q_{i j}, i, j \in S\right)$ given by

$$
\begin{aligned}
& q_{i, i+1}=\lambda_{i}, \quad q_{i+1, i}=\mu_{i+1}, \quad q_{i i}=-\left(\lambda_{i}+\mu_{i}+\gamma_{i}\right), \quad i \geq 0, \\
& q_{i j}=0, \quad|i-j|>1,
\end{aligned}
$$

where $\lambda_{i}>0$ and $\gamma_{i} \geq 0$ for $i \geq 0, \mu_{i}>0$ for $i>0$, and $\mu_{0}=0$. The parameters $\lambda_{i}$ and $\mu_{i}$ are the birth and death rates in state $i$, while $\gamma_{i}$ may be regarded as the rate of absorption, or killing, into a fictitious state $\partial$, say. A transition to the absorbing state is sometimes referred to in the literature as a total catastrophe (see for example Chao and Zheng (2003)).

We will assume that the (standard) transition function $P(.) \equiv\left\{p_{i j}(), i,. j \in\right.$ $S$ \}, where

$$
p_{i j}(t) \equiv \operatorname{Pr}\{X(t)=j \mid X(0)=i\}, \quad t \geq 0, i, j \in S,
$$

is the minimal $Q$-function, that is, the minimal transition function with $q$ matrix $Q$. As a consequence $P($.) satisfies the system

$$
P^{\prime}(t)=Q P(t)=P(t) Q, \quad t \geq 0,
$$

of backward and forward equations (see for example Anderson (1991)). We note parenthetically that imposing the forward equations is equivalent to imposing "continuity at infinity", in the sense that almost surely the only discontinuities of the process (before absorption) are simple discontinuities with saltus \pm 1 , even if the process goes to infinity in finite time and returns from there (see Karlin and McGregor (1959)). A prominent role in what follows will be played by the polynomials $\left\{R_{n}\right\}$ which are uniquely determined by the transition rates of $\mathcal{X}$ through the recurrence relation

$$
\begin{aligned}
& \lambda_{n} R_{n+1}(x)=\left(\lambda_{n}+\mu_{n}+\gamma_{n}-x\right) R_{n}(x)-\mu_{n} R_{n-1}(x), \quad n \geq 1, \\
& \lambda_{0} R_{1}(x)=\lambda_{0}+\gamma_{0}-x, \quad R_{0}(x)=1 .
\end{aligned}
$$

Karlin and McGregor (1957) have shown that if the killing rates $\gamma_{i}$ are all zero except $\gamma_{0} \geq 0$ (in their notation $\gamma_{i}=0$ for all $i$, but $\mu_{0} \geq 0$, and the 
absorbing state is labeled -1$)$, then the transition probabilities $p_{i j}(t)$ may be represented in the form

$$
p_{i j}(t)=\pi_{j} \int_{0}^{\infty} e^{-x t} R_{i}(x) R_{j}(x) \psi(d x), \quad t \geq 0, i, j \in S .
$$

Here $\pi_{n}$ are constants given by

$$
\pi_{0} \equiv 1 \text { and } \pi_{n} \equiv \frac{\lambda_{0} \lambda_{1} \ldots \lambda_{n-1}}{\mu_{1} \mu_{2} \ldots \mu_{n}}, \quad n>0,
$$

and $\psi$ is a Borel measure of total mass 1 on $[0, \infty)$ with respect to which the polynomials $\left\{R_{n}\right\}$ are orthogonal. The orthogonalizing measure for $\left\{R_{n}\right\}$ is in virtual all practical cases unique, and in many cases known explicitly. The usefulness of the integral representation (4) derives from the monotonic properties of $e^{-x t}$, and from the fact that the dependence on $t, i$ and $j$ is factored in the integrand.

If we allow for killing rates $\gamma_{i} \geq 0$ in each state $i \in S$, the polynomials $\left\{R_{n}\right\}$ of (3) still constitute an orthogonal polynomial sequence with respect to a Borel measure $\psi$ on $[0, \infty)$. The main purpose of this note is to point out that also the representation (4) remains valid in this case.

The remainder of this paper is organized as follows. In Section 2 we will gather some preliminary results, which are needed in Section 3 to prove our assertion. In Section 4 we will investigate under which condition on $Q$ the minimal $Q$-function $P($.$) is the only Q$-function satisfying both backward and forward equations. We conclude in Section 5 with an example.

\section{An associated birth-death process}

We shall have use for the quantities $\tilde{\lambda}_{i}$ and $\tilde{\mu}_{i}, i \geq 0$, recurrently defined by

$$
\begin{aligned}
& \tilde{\mu}_{0}=0, \quad \tilde{\lambda}_{0}=\lambda_{0}+\gamma_{0} \\
& \tilde{\mu}_{i}=\left(\lambda_{i-1} / \tilde{\lambda}_{i-1}\right) \mu_{i}, \quad \tilde{\lambda}_{i}=\lambda_{i}+\gamma_{i}+\mu_{i}-\tilde{\mu}_{i}, \quad i>0 .
\end{aligned}
$$

It is easily seen by induction that

$$
\tilde{\lambda}_{i} \geq \lambda_{i}+\gamma_{i} \geq \lambda_{i}>0 \text { and } \tilde{\mu}_{i+1}>0, \quad i \geq 0,
$$


so that $\tilde{\lambda}_{i}$ and $\tilde{\mu}_{i}$ may be interpreted as the birth and death rates, respectively, of a (conservative) birth-death process $\tilde{\mathcal{X}} \equiv\{\tilde{X}(t), t \geq 0\}$ on $S$ with $q$-matrix $\tilde{Q} \equiv\left(\tilde{q}_{i j}\right)$ given by

$$
\begin{aligned}
& \tilde{q}_{i, i+1}=\tilde{\lambda}_{i}, \quad \tilde{q}_{i+1, i}=\tilde{\mu}_{i+1}, \quad \tilde{q}_{i i}=-\left(\tilde{\lambda}_{i}+\tilde{\mu}_{i}\right), \quad i \geq 0, \\
& \tilde{q}_{i j}=0, \quad|i-j|>1 .
\end{aligned}
$$

We let

$$
\tilde{\pi}_{0} \equiv 1 \text { and } \tilde{\pi}_{n} \equiv \frac{\tilde{\lambda}_{0} \tilde{\lambda}_{1} \ldots \tilde{\lambda}_{n-1}}{\tilde{\mu}_{1} \tilde{\mu}_{2} \ldots \tilde{\mu}_{n}}, \quad n>0,
$$

and observe from (1), (6) and (7) that

$$
\tilde{\Pi}^{1 / 2} \tilde{Q} \tilde{\Pi}^{-1 / 2}=\Pi^{1 / 2} Q \Pi^{-1 / 2}
$$

where $\tilde{\Pi}^{1 / 2}$ and $\Pi^{1 / 2}$ denote the diagonal matrices with entries $\sqrt{\tilde{\pi}_{n}}$ and $\sqrt{\pi_{n}}$, respectively, on the diagonals.

Evidently, $\tilde{\mathcal{X}}$ need not be uniquely determined by $\tilde{Q}$, but in what follows we will assume that $\tilde{\mathcal{X}}$ is the minimal $\tilde{Q}$-process, represented by the minimal $\tilde{Q}$-function $\tilde{P}(.) \equiv\left\{\tilde{p}_{i j}(), i,. j \in S\right\}$, which therefore satisfies the system

$$
\tilde{P}^{\prime}(t)=\tilde{Q} \tilde{P}(t)=\tilde{P}(t) \tilde{Q}, \quad t \geq 0
$$

of backward and forward equations. Also, Karlin and McGregor's integral representation applies to $\tilde{p}_{i j}(t)$, that is,

$$
\tilde{p}_{i j}(t)=\tilde{\pi}_{j} \int_{0}^{\infty} e^{-x t} \tilde{R}_{i}(x) \tilde{R}_{j}(x) \tilde{\psi}(d x), \quad t \geq 0, i, j \in S,
$$

where $\left\{\tilde{R}_{n}\right\}$ are the polynomials satisfying the recurrence

$$
\begin{aligned}
& \tilde{\lambda}_{n} \tilde{R}_{n+1}(x)=\left(\tilde{\lambda}_{n}+\tilde{\mu}_{n}-x\right) \tilde{R}_{n}(x)-\tilde{\mu}_{n} \tilde{R}_{n-1}(x), \quad n \geq 1, \\
& \tilde{\lambda}_{0} \tilde{R}_{1}(x)=\tilde{\lambda}_{0}-x, \quad \tilde{R}_{0}(x)=1,
\end{aligned}
$$

and $\tilde{\psi}$ is a Borel measure (of total mass 1 ) on $[0, \infty)$ with respect to which the $\left\{\tilde{R}_{n}\right\}$ are orthogonal. Actually, if $\tilde{Q}$ is such that

$$
\sum_{n=0}^{\infty}\left(\tilde{\pi}_{n}+\frac{1}{\tilde{\lambda}_{n} \tilde{\pi}_{n}}\right)=\infty
$$

then the Stieltjes moment problem associated with $\left\{\tilde{R}_{n}\right\}$ is determined, which means that $\tilde{\psi}$ is the unique orthogonalizing measure on $[0, \infty)$ for $\left\{\tilde{R}_{n}\right\}$. In this 
case $\tilde{P}($.$) is the unique \tilde{Q}$-function satisfying the system (10) of backward and forward equations. If the series in (13) converges, then the Stieltjes moment problem is indeterminate, that is, there are infinitely many orthogonalizing measures for $\left\{\tilde{R}_{n}\right\}$. In this case there are also infinitely many $\tilde{Q}$-functions satisfying (10). The measure $\tilde{\psi}$ corresponding to the minimal $\tilde{Q}$-function may now be characterized as the one which is supported by the zeros of the (entire) function $\tilde{R}_{\infty}(x) \equiv \lim _{n \rightarrow \infty} \tilde{R}_{n}(x)$; we will refer to $\tilde{\psi}$ as the minimal measure for $\left\{\tilde{R}_{n}\right\}$ (see Karlin and McGregor (1957) for more details).

It is sometimes desirable for $\tilde{\mathcal{X}}$ to be non-explosive, which requires $\tilde{Q}$ to be such that

$$
\sum_{n=0}^{\infty} \frac{1}{\tilde{\lambda}_{n} \tilde{\pi}_{n}} \sum_{i=0}^{n} \tilde{\pi}_{i}=\infty,
$$

which is stronger than (13). If (and only if) condition (14) is satisfied, then the minimal $\tilde{Q}$-function $\tilde{P}($.) is in fact the unique $\tilde{Q}$-function satisfying just the backward equations $\tilde{P}^{\prime}(t)=\tilde{Q} \tilde{P}(t)$.

We conclude this section with the observation that, apart from a multiplicative constant, the polynomials $\tilde{R}_{n}$ and $R_{n}$ are identical. Indeed, it follows readily by induction from (6) and the recurrence relations (3) and (12) that

$$
\tilde{R}_{n}(x)=\frac{\lambda_{0} \lambda_{1} \ldots \lambda_{n-1}}{\tilde{\lambda}_{0} \tilde{\lambda}_{1} \ldots \tilde{\lambda}_{n-1}} R_{n}(x)=\sqrt{\frac{\pi_{n}}{\tilde{\pi}_{n}}} R_{n}(x), \quad n \geq 0 .
$$

So, the polynomials $\left\{R_{n}\right\}$ of (3) are orthogonal with respect to any measure which is an orthogonalizing measure for $\left\{\tilde{R}_{n}\right\}$, and with respect to $\tilde{\psi}$ in particular.

\section{Representation}

Our main result is expressed in the corollary to the following theorem.

Theorem 1 The minimal $Q$-function $P(.) \equiv\left\{p_{i j}().\right\}$ and the minimal $\tilde{Q}$ function $\tilde{P}(.) \equiv\left\{\tilde{p}_{i j}().\right\}$ satisfy

$$
P(t)=\Pi^{-1 / 2} \tilde{\Pi}^{1 / 2} \tilde{P}(t) \tilde{\Pi}^{-1 / 2} \Pi^{1 / 2}, \quad t \geq 0 .
$$


Proof Denoting the right-hand side of (16) by $F(t)$, it follows from (9) and (10) that $F($.$) satisfies the system of backward and forward equations (2). F($.) is also the minimal non-negative solution of (2) since the opposite would imply that $\tilde{P}($.$) is not the minimal non-negative solution of (10), and therefore (see for$ example Anderson, 1991, Theorem 2.2.2) not the minimal $\tilde{Q}$-function. Hence, by the same theorem in Anderson (1991), $F($.$) is the minimal Q$-function, that is, $F(t)=P(t), t \geq 0$.

Corollary 2 The minimal $Q$-function $P(.) \equiv\left\{p_{i j}().\right\}$ of the process $\mathcal{X}$ may be represented in the form (4), where $\left\{R_{n}\right\}$ are the polynomials defined in (3) and $\psi$ is the orthogonalizing measure (of total mass 1) for $\left\{R_{n}\right\}$ on $[0, \infty)$ which, if it is not unique, is the minimal measure.

Proof By (16) and (11) we have

$$
p_{i j}(t)=\sqrt{\frac{\tilde{\pi}_{i} \pi_{j}}{\pi_{i} \tilde{\pi}_{j}}} \tilde{p}_{i j}(t)=\sqrt{\frac{\tilde{\pi}_{i}}{\pi_{i}} \pi_{j} \tilde{\pi}_{j}} \int_{0}^{\infty} e^{-x t} \tilde{R}_{i}(x) \tilde{R}_{j}(x) \tilde{\psi}(d x) .
$$

Substituting (15) and noting, in view of (15), that $\left\{R_{n}\right\}$ is orthogonal with respect to $\psi=\tilde{\psi}$ yields the result.

So, as announced, we may conclude that the representation (4) remains valid whether the killing rates $\gamma_{i}, i>0$, are zero or not.

As in Karlin and McGregor (1957), certain non-minimal $Q$-functions (if there are any) may also be represented in the form (4), with $\psi$ replaced by an appropriate (non-minimal) orthogonalizing measure for $\left\{R_{n}\right\}$. A necessary and sufficient condition for $P($.) to be the only $Q$-function satisfying (2) will be derived in the next section, but otherwise we will not pursue this issue.

As an aside we note that the concept of similarity for birth-death processes introduced in Lenin et al. (2000) may be extended to birth-death processes with killing. Namely, in view of (16) the transition probability functions $p_{i j}($.$) and$ $\tilde{p}_{i j}($.$) differ only by a multiplicative constant. Hence the process \mathcal{X}$ is similar (in the sense of Lenin et al. (2000)) to the pure birth-death process $\tilde{\mathcal{X}}$. 


\section{Uniqueness}

If condition (13) is satisfied then the minimal $\tilde{Q}$-function $\tilde{P}($.$) is the unique$ $\tilde{Q}$-function satisfying (10), and, as we shall see in this section, $P($.$) is the$ unique $Q$-function satisfying (2). On the other hand, (13) is not necessary for uniqueness of $P($.$) . Before giving a necessary and sufficient condition we need$ some preliminary results.

First, we observe from (15) and (6) that for all $x$

$$
\tilde{\lambda}_{n} \tilde{\pi}_{n} \tilde{R}_{n}(x) \tilde{R}_{n+1}(x)=\lambda_{n} \pi_{n} R_{n}(x) R_{n+1}(x), \quad n \geq 0 .
$$

It is easily seen from (12) that $\tilde{R}_{n}(0)=1$ for all $n$, so, as a consequence of (15) and (17), we have

$$
\tilde{\pi}_{n}=\pi_{n} R_{n}^{2}(0), \quad n \geq 0,
$$

and

$$
\tilde{\lambda}_{n} \tilde{\pi}_{n}=\lambda_{n} \pi_{n} R_{n}(0) R_{n+1}(0), \quad n \geq 0 .
$$

It will also be useful to note from the recurrence relation (3) and the fact that $\lambda_{n-1} \pi_{n-1}=\mu_{n} \pi_{n}$ that, for $n \geq 1$,

$$
\lambda_{n} \pi_{n}\left(R_{n+1}(0)-R_{n}(0)\right)=\lambda_{n-1} \pi_{n-1}\left(R_{n}(0)-R_{n-1}(0)\right)+\gamma_{n} \pi_{n} R_{n}(0),
$$

while $\lambda_{0} \pi_{0}\left(R_{1}(0)-R_{0}(0)\right)=\gamma_{0} \pi_{0} R_{0}(0)$, so that

$$
\lambda_{n} \pi_{n}\left(R_{n+1}(0)-R_{n}(0)\right)=\sum_{k=0}^{n} \gamma_{k} \pi_{k} R_{k}(0), \quad n \geq 0,
$$

and

$$
R_{n+1}(0)=1+\sum_{j=0}^{n} \frac{1}{\lambda_{j} \pi_{j}} \sum_{k=0}^{j} \gamma_{k} \pi_{k} R_{k}(0), \quad n \geq 0 .
$$

Our final preliminary result is the following lemma, which is the generalization to the setting at hand of (part of) Lemma 6 (on p. 526) of Karlin and McGregor (1957). Recall that $\psi(=\tilde{\psi})$ is either the unique or else the minimal orthogonalizing measure for $\left\{\tilde{R}_{n}\right\}$, and hence for $\left\{R_{n}\right\}$. 
Lemma 3 We have

$$
\sum_{j=0}^{\infty} \gamma_{j} \pi_{j} \int_{0}^{\infty} x^{-1} R_{j}(x) \psi(d x)=1-\lim _{n \rightarrow \infty} \frac{1}{R_{n}(0)} .
$$

Proof From Karlin and McGregor, 1957, Lemma 6 we know that

$$
\int_{0}^{\infty} x^{-1} \tilde{\psi}(d x)=\sum_{n=0}^{\infty} \frac{1}{\tilde{\lambda}_{n} \tilde{\pi}_{n}},
$$

where both members may be infinite. It subsequently follows by induction from the recurrence relation (12) that

$$
\int_{0}^{\infty} x^{-1} \tilde{R}_{j}(x) \tilde{\psi}(d x)=\sum_{n=j}^{\infty} \frac{1}{\tilde{\lambda}_{n} \tilde{\pi}_{n}}, \quad j \geq 0 .
$$

Hence, with the help of (15), (19), (18), and (20) we can write

$$
\begin{aligned}
\sum_{j=0}^{\infty} \gamma_{j} \pi_{j} \int_{0}^{\infty} x^{-1} R_{j}(x) \psi(d x) & =\sum_{j=0}^{\infty} \gamma_{j} \sqrt{\pi_{j} \tilde{\pi}_{j}} \int_{0}^{\infty} x^{-1} \tilde{R}_{j}(x) \tilde{\psi}(d x) \\
& =\sum_{j=0}^{\infty} \gamma_{j} \sqrt{\pi_{j} \tilde{\pi}_{j}} \sum_{n=j}^{\infty} \frac{1}{\tilde{\lambda}_{n} \tilde{\pi}_{n}} \\
& =\sum_{j=0}^{\infty} \gamma_{j} \pi_{j} R_{j}(0) \sum_{n=j}^{\infty} \frac{1}{\lambda_{n} \pi_{n} R_{n}(0) R_{n+1}(0)} \\
& =\sum_{n=0}^{\infty} \frac{1}{\lambda_{n} \pi_{n} R_{n}(0) R_{n+1}(0)} \sum_{j=0}^{n} \gamma_{j} \pi_{j} R_{j}(0) \\
& =\sum_{n=0}^{\infty}\left(\frac{1}{R_{n}(0)}-\frac{1}{R_{n+1}(0)}\right),
\end{aligned}
$$

which yields the required result.

We can now give a necessary and sufficient condition for $P($.$) , the minimal$ $Q$-function, to be the unique $Q$-function satisfying (2).

Theorem 4 In order that there be only one $Q$-function $P($.$) satisfying the com-$ bined system of backward and forward equations it is necessary and sufficient that at least one of the two conditions

$$
\lim _{n \rightarrow \infty} R_{n}(0)=\infty
$$


or

$$
\sum_{n=0}^{\infty}\left(\pi_{n}+\frac{1}{\lambda_{n} \pi_{n}}\right)=\infty
$$

be satisfied.

Proof As in the proof of Theorem 15 of Karlin and McGregor (1957), we consider the Laplace transform $D(s) \equiv\left(D_{i j}(s)\right)$ of the difference of any two $Q$-functions satisfying (2) and find that

$$
D_{i j}(s)=\pi_{j} R_{i}(-s) R_{j}(-s) D_{00}(s), \quad s \geq 0 .
$$

Since the zeros of $\tilde{R}_{n}(x)$, and hence $R_{n}(x)$, are all positive (see Karlin and McGregor (1957)), while, by (21), $R_{n}(0)$ is increasing in $n$, we have

$$
R_{n}(-s) \geq R_{n}(0) \geq 1, \quad s \geq 0,
$$

for all $n$. Hence, if (24) is satisfied then $R_{i}(-s) \rightarrow \infty$ as $i \rightarrow \infty$. But since $D_{i j}(s)$ is bounded we must have $D_{00}(s)=0$, and hence $D_{i j}(s)=0$ for all $i, j$. On the other hand, if (24) is not satisfied then $\sum_{j}\left(\lambda_{j} \pi_{j}\right)^{-1}<\infty$ as a consequence of (21). So if, at the same time, (25) is satisfied, we must have $\sum_{j} \pi_{j}=\infty$, and hence $\sum_{j} \pi_{j} R_{j}(-s)=\infty$ for $s \geq 0$. But, since $\sum_{j} D_{i j}(s)$ must be bounded, it follows again that $D_{00}(s)=0$, and hence $D_{i j}(s)=0$ for all $i, j$.

Now suppose neither (24) nor (25) are satisfied. Then, in view of (18) and (19), also (13) is not satisfied. As a result there is a number $\xi_{1}>0$ and a one-parameter family $\left\{\psi_{\xi}, 0 \leq \xi \leq \xi_{1}\right\}$ of so-called extremal solutions to the Stieltjes moment problem associated with $\left\{\tilde{R}_{n}\right\}$. The index $\xi$ in $\psi_{\xi}$ denotes the smallest point in the support of the measure, and the minimal measure $\psi=\tilde{\psi}$ should be identified with $\psi_{\xi_{1}}$ (see Karlin and McGregor (1957) or van Doorn (1987) for more details). Next letting

$$
\phi(\xi) \equiv \sum_{j=0}^{\infty} \gamma_{j} \pi_{j} \int_{0}^{\infty} x^{-1} R_{j}(x) \psi_{\xi}(d x), \quad 0 \leq \xi \leq \xi_{1},
$$

we note that, by Lemma 3 , the boundedness of $R_{n}(0)$ amounts to $\phi\left(\xi_{1}\right)<1$. The proof of Theorem 15 of Karlin and McGregor (1957) can now be copied to show that $\phi(\xi)$ is continuous, so that there must be a number $\xi_{0}$ such that 
$\phi(\xi)<1$ for $\xi \in\left(\xi_{0}, \xi_{1}\right]$. As in Karlin and McGregor (1957) the extremal measures $\psi_{\xi}, \xi_{0}<\xi \leq \xi_{1}$, can subsequently be used to construct infinitely many distinct $Q$-functions satisfying (2).

If only finitely many $\gamma_{i}$ are positive then, by (21), statement (24) is equivalent to $\sum_{n=0}^{\infty}\left(\lambda_{n} \pi_{n}\right)^{-1}=\infty$, so that (25) alone is necessary and sufficient for uniqueness. Thus we have regained the necessary and sufficient condition for uniqueness given in Theorem 15 of Karlin and McGregor (1957) in the case that killing is possible in state 0 only.

In general, the first condition in the above theorem does not imply the second one. Indeed, from (19) we obtain

$$
R_{n+1}(0)=R_{n}(0)+\frac{\gamma_{n}}{\lambda_{n}} R_{n}(0)+\frac{1}{\lambda_{n} \pi_{n}} \sum_{k=0}^{n-1} \gamma_{k} \pi_{k} R_{k}(0) .
$$

Hence, we may choose $\lambda_{n}$ and $\mu_{n}$ such that the series in (25) converges, and subsequently $\gamma_{n}$ successively so large that $R_{n+1}(0) \geq R_{n}(0)+1$. As a result (24) does hold.

We also note that if $R_{n}(0)$ is bounded then (13) and (25) are equivalent, in view of (18) and (19). So, in accordance with our assertion at the beginning of this section, if $\tilde{P}($.$) is the unique \tilde{Q}$-function satisfying (10), then $P($.$) is the$ unique $Q$-function satisfying (2). (The reverse does not necessarily hold true.)

The integral in (22) seems enigmatic, but has in fact a clear probabilistic interpretation. Namely, let $T_{\partial}$ denote the killing time, that is, the (possibly defective) random variable representing the time at which absorption in the absorbing state $\partial$ occurs. Since, by the forward equations,

$$
\operatorname{Pr}\left\{T_{\partial} \leq t \mid X(0)=0\right\}=\sum_{j=0}^{\infty} \gamma_{j} \int_{0}^{t} p_{0 j}(u) d u,
$$

we have

$$
\operatorname{Pr}\left\{T_{\partial}<\infty \mid X(0)=0\right\}=\sum_{j=0}^{\infty} \gamma_{j} \int_{0}^{\infty} p_{0 j}(u) d u,
$$

so that, by substituting the integral representation (4) and interchanging the integrals, we get

$$
\operatorname{Pr}\left\{T_{\partial}<\infty \mid X(0)=0\right\}=\sum_{j=0}^{\infty} \gamma_{j} \pi_{j} \int_{0}^{\infty} x^{-1} R_{j}(x) \psi(d x) .
$$


So Lemma 3 tells us that absorption at $\partial$ from state 0 (and hence from any state) is certain if and only if $R_{n}(0)$ is unbounded. More information about the killing time $T_{\partial}$ is given in van Doorn and Zeifman (2005).

\section{Example}

If the killing rates are constant, $\gamma_{i}=\gamma$, say, then, by conditioning, the transition probabilities $p_{i j}($.$) of the process with killing can simply be expressed in terms$ of the transition probabilities $\tilde{p}_{i j}($.$) of the process with the same birth and death$ rates, but zero killing rates. Namely,

$$
p_{i j}(t)=e^{-\gamma t} \tilde{p}_{i j}(t), \quad i, j \in S, t \geq 0 .
$$

So interesting cases arise when the killing rates are state dependent.

As an example we will consider the process $\mathcal{X}$ with linear birth, death and killing rates, namely,

$$
\lambda_{i}=\lambda i+\theta, \quad \mu_{i}=i \mu, \quad \gamma_{i}=i \gamma, \quad i \in S,
$$

with $\lambda, \theta, \mu, \gamma>0$. It is easy to see that (25) is satisfied, so $\mathcal{X}$ is uniquely determined by its rates. Karlin and Tavaré (1982) have analysed the process by adroitly relating it to an honest birth-death process with known transition probabilities. We shall see that a direct approach based on the integral representation (4) yields the same result. Indeed, the recurrence relation (3) becomes

$$
\begin{aligned}
& (\lambda n+\theta) R_{n+1}(x)=(\theta+(\lambda+\mu+\gamma) n-x) R_{n}(x)-\mu n R_{n-1}(x), \quad n>1, \\
& \theta R_{1}(x)=\theta-x, \quad R_{0}(x)=1 .
\end{aligned}
$$

Now writing

$$
\beta \equiv \frac{\theta}{\lambda}, \quad \rho \equiv \sqrt{(\lambda+\mu+\gamma)^{2}-4 \lambda \mu}, \quad \kappa \equiv \theta\left(1-\frac{2 \mu}{\lambda+\mu+\gamma+\rho}\right)
$$

and

$$
P_{n}(x) \equiv\left(\frac{2 \lambda}{\lambda+\mu+\gamma-\rho}\right)^{n}(\beta)_{n} R_{n}(\rho x+\kappa), \quad n \geq 0,
$$

where $(\beta)_{n} \equiv \Gamma(\beta+n) / \Gamma(\beta)$, we see after a little algebra that $\left\{P_{n}\right\}$ satisfies the recurrence

$$
c P_{n+1}(x)=((c-1) x+(c+1) n+c \beta) P_{n}(x)-n(n+\beta-1) P_{n-1}(x),
$$


where

$$
c=\frac{\lambda+\mu+\gamma-\rho}{\lambda+\mu+\gamma+\rho} .
$$

The polynomials $P_{n}$ can now be identified (see Chihara, 1978, Section VI.3) with the Meixner polynomials of the first kind, which are orthogonal with respect to a discrete measure with masses at $0,1, \ldots$ Specifically,

$$
(1-c)^{\beta} \sum_{x=0}^{\infty} P_{m}(x) P_{n}(x) \frac{c^{x}(\beta)_{x}}{x !}=\delta_{m, n} \frac{(\beta)_{n} n !}{c^{n}}, \quad m, n \geq 0 .
$$

As a consequence the orthogonality relation for the polynomials $\left\{R_{n}\right\}$ may be given as

$$
(1-c)^{\beta} \sum_{x=0}^{\infty} R_{m}(\rho x+\kappa) R_{n}(\rho x+\kappa) \frac{c^{x}(\beta)_{x}}{x !}=\delta_{m, n} \frac{\mu^{n} n !}{(\beta)_{n} \lambda^{n}}, \quad m, n \geq 0 .
$$

By elementary substitution of these findings in the integral representation (4) we regain the result obtained earlier in Karlin and Tavaré (1982). 


\section{References}

Anderson, W.J. (1991), Continuous-Time Markov Chains (Springer, New York).

Chao, X. and Zheng, Y. (2003), Transient analysis of immigration birth-death processes with total catastrophes, Probab. Engrg. Inform. Sci. 17, 83-106.

Chihara, T.S. (1978), An Introduction to Orthogonal Polynomials (Gordon and Breach, New York).

van Doorn, E.A. (1987), The indeterminate rate problem for birth-death processes, Pacific J. Math. 130, 379-393.

van Doorn, E.A. and Zeifman, A.I. (2005), Extinction probability in a birthdeath process with killing, J. Appl. Probab. 42, to appear.

Karlin, S. and McGregor, J.L. (1957), The differential equations of birth-anddeath processes, and the Stieltjes moment problem, Trans. Amer. Math. Soc. 85, 589-646.

Karlin, S. and McGregor, J.L. (1959), A characterization of birth and death processes, Proc. Natl. Acad. Sci. USA 45, 375-379.

Karlin, S. and Tavaré, S. (1982), Linear birth and death processes with killing, J. Appl. Probab. 19, 477-487.

Lenin, R.B., Parthasarathy, P.R., Scheinhardt, W.R.W. and van Doorn, E.A. (2000), Families of birth-death processes with similar time-dependent behaviour. J. Appl. Probab. 37, 835-849. 